\title{
THE NINTH DATA RELEASE OF THE SLOAN DIGITAL SKY SURVEY: FIRST SPECTROSCOPIC DATA FROM THE SDSS-III BARYON OSCILLATION SPECTROSCOPIC SURVEY
}

Christopher P. Ahn ${ }^{1}$, Rachael Alexandrofr ${ }^{2}$, Carlos Allende Prieto ${ }^{3,4}$, Scott F. Anderson $^{5}$, Timothy Anderton ${ }^{1}$, Brett H. Andrews ${ }^{6}$, Éric Aubourg ${ }^{7}$, Stephen Bailey $^{8}$, Eduardo Balbinot ${ }^{9}, 10$, Rory Barnes ${ }^{5}$, Julian Bautista ${ }^{7}$, Timothy C. Beers ${ }^{11,12}$, Alessandra Beifiori ${ }^{13}$, Andreas A. Berlind ${ }^{14}$, Vaishali Bhardwaj ${ }^{5}$, Dmitry Bizyaev ${ }^{15}$, Cullen H. Blake ${ }^{2}$, Michael R. Blanton ${ }^{16}$, Michael Blomevist ${ }^{17}$, John J. Bochanski ${ }^{5,18}$, Adam S. Bolton ${ }^{1}$, Arnaud Borde ${ }^{19}$, Jo Bovy ${ }^{20,95}$, W. N. Brandt ${ }^{21,22}$, J. Brinkmann ${ }^{15}$, Peter J. Brown ${ }^{1,23}$, Joel R. Brownstein ${ }^{1}$, Kevin Bundy $^{24}$, N. G. Busca ${ }^{7}$, William Carithers ${ }^{8}$, Aurelio R. Carnero ${ }^{10,25}$, Michael A. Carr ${ }^{2}$, Dana I. Casetti-Dinescu ${ }^{26}$, Yanmei Chen $^{27,28}$, Cristina Chiappini ${ }^{10,29}$, Johan Comparat $^{30}$, Natalia Connolly $^{31}$, Justin R. Crepp ${ }^{32,33}$, Stefano Cristiani ${ }^{34,35}$, Rupert A. C. Croft ${ }^{36}$, Antonio J. Cuesta ${ }^{37}$, Luiz N. Da Costa ${ }^{10,25}$, James R. A. Davenport ${ }^{5}$, Kyle S. Dawson ${ }^{1}$, Roland de Putter ${ }^{38,39}$, Nathan De LeE ${ }^{14}$, Timothée Delubac ${ }^{19}$, Saurav Dhital ${ }^{14,40}$, Anne Ealet ${ }^{41}$, Garrett L. Ebelke ${ }^{15,42}$, Edward M. Edmondson ${ }^{43}$, Daniel J. Eisenstein ${ }^{44}$,

S. Escoffier ${ }^{41}$, Massimiliano Esposito ${ }^{3,4}$, Michael L. Evans ${ }^{5}$, Xiaohui Fan ${ }^{45}$, Bruno Femenía Castellá ${ }^{3,4}$, Emma Fernández Alvar ${ }^{3,4}$, Leticia D. Ferreira ${ }^{10,46}$, N. Filiz AK ${ }^{21,22,47}$, Hayley Finley $^{48}$, Scott W. Fleming ${ }^{21,49,50,}$ Andreu Font-Ribera ${ }^{8,51}$, Peter M. Frinchaboy ${ }^{52}$, D. A. García-Hernández ${ }^{3,4}$, A. E. García Pérez ${ }^{53}$, Jian Ge ${ }^{49}$, R. Génova-Santos ${ }^{3,4}$, Bruce A. Gillespie ${ }^{15}$, Léo Girardi ${ }^{10,54}$, Jonay I. GonZález Hernández ${ }^{3}$, Eva K. Grebel ${ }^{55}$, James E. Gunn ${ }^{2}$, Hong Guo ${ }^{56}$, Daryl Haggard ${ }^{57}$, Jean-Christophe Hamilton ${ }^{7}$, David W. Harris ${ }^{1}$, Suzanne L. Hawley ${ }^{5}$, Frederick R. Hearty ${ }^{53}$, Shirley Ho ${ }^{36}$, David W. HogG ${ }^{16}$, Jon A. Holtzman ${ }^{42}$, Klaus Honscheid ${ }^{58}$, J. HuehnerhofF ${ }^{15}$,

IneSE I. IVANS ${ }^{1}, \breve{Z ̌ E L J K O ~ I V E Z I C ́}^{5,59,60}$, HeATHER R. JACOBSON ${ }^{61,96}$, LINHUA JiANG ${ }^{45}$, JONAS JOHANSSON ${ }^{43,62}$,

Jennifer A. Johnson ${ }^{6}$, Guinevere Kauffmann ${ }^{62}$, David Kirkby ${ }^{17}$, Jessica A. KirkPatrick ${ }^{8,63}$, Mark A. Klaene ${ }^{15}$, Gillian R. KnapP ${ }^{2}$, Jean-Paul Kneib ${ }^{30}$, Jean-Marc Le GofF ${ }^{19}$, Alexie Leauthaud ${ }^{24}$, Khee-Gan LeE ${ }^{64}$, Young Sun LeE ${ }^{12}$, Daniel C. Long ${ }^{15}$, Craig P. Loomis ${ }^{2}$, Sara Lucatello ${ }^{54}$, Britt Lundgren ${ }^{37}$, Robert H. Lupton ${ }^{2}$,

Bo MA ${ }^{49}$, Zhibo Ma ${ }^{56}$, Nicholas MacDonald ${ }^{5}$, Claude E. Mack III $^{14}$, Suvrath Mahadevan ${ }^{21,50}$, Marcio A. G. Maia ${ }^{10,25}$, Steven R. Majewski ${ }^{53}$, Martin MaKleR ${ }^{10,65}$, Elena Malanushenko ${ }^{15,42}$, Viktor Malanushenko ${ }^{15,42}$, A. Manchado ${ }^{3,4}$,

Rachel Mandelbaum ${ }^{2,36}$, Marc Manera ${ }^{43}$, Claudia Maraston ${ }^{43}$, Daniel Margala ${ }^{17}$, Sarah L. Martell ${ }^{55,66}$, Cameron K. McBride ${ }^{44}$, Ian D. McGreer ${ }^{45}$, Richard G. McMahon ${ }^{67,68}$, Brice Ménard ${ }^{24,69}$, Sz. Meszaros ${ }^{3,4}$,

Jordi Miralda-Escudét ${ }^{38,70}$, Antonio D. Montero-Dorta ${ }^{1,71}$, Francesco Montesano ${ }^{13}$, Heather L. Morrison ${ }^{56}$, Demitri Muna ${ }^{16}$, Jeffrey A. Munn ${ }^{72}$, Hitoshi Murayama ${ }^{24}$, Adam D. Myers ${ }^{73}$, A. F. Neto ${ }^{10}$, Duy Cuong Nguyen ${ }^{49,74}$, Robert C. Nichol ${ }^{43}$, David L. Nidever ${ }^{53}$, Pasquier Noterdaeme ${ }^{48}$, Sebastián E. Nuza ${ }^{29}$, Ricardo L. C. Ogando ${ }^{10,25}$,

Matthew D. Olmstead ${ }^{1}$, Daniel J. Oravetz ${ }^{15}$, Russell Owen $^{5}$, Nikhil Padmanabhan ${ }^{37}$,

Nathalie Palanque-Delabrouille ${ }^{19}$, Kaike Pan ${ }^{15}$, John K. Parejko ${ }^{37}$, Prachi Parihar ${ }^{2}$, Isabelle Pâris ${ }^{48,75}$, Petchara Pattarakijwanich ${ }^{2}$, Joshua Pepper ${ }^{14}$, Will J. Percival ${ }^{43}$, Ismael Pérez-Fournon ${ }^{3,4}$, Ignasi Pérez-Ràfols ${ }^{38}$, Patrick Petitjean ${ }^{48}$, Janine Pforr ${ }^{11,43}$, Matthew M. Pieri ${ }^{43}$, Marc H. Pinsonneault ${ }^{6}$, G. F. Porto de Mello ${ }^{10,46}$,

Francisco Prada $^{71,76,77}$, Adrian M. Price-Whelan ${ }^{78}$, M. Jordan Raddick ${ }^{69}$, RAFael Rebolo $^{3,79}$, James Rich ${ }^{19}$,

Gordon T. Richards ${ }^{80}$, Annie C. Robin ${ }^{81}$, Helio J. Rocha-Pinto ${ }^{10,46}$, Constance M. Rockosi ${ }^{82}$, Natalie A. Roe ${ }^{8}$,

Ashley J. Ross ${ }^{43}$, Nicholas P. Ross ${ }^{8}$, Graziano Rossi ${ }^{19,83}$, J. A. Rubiño-Martin ${ }^{3,4}$, Lado Samushia ${ }^{43,84}$,

J. Sanchez Almeida ${ }^{3,4}$, Ariel G. Sánchez ${ }^{13}$, Basílio Santiago ${ }^{9,10}$, Conor SaYres ${ }^{5}$, David J. Schlegel ${ }^{8}$,

Katharine J. Schlesinger ${ }^{6,82}$, Sarah J. Schmidt ${ }^{5}$, Donald P. Schneider ${ }^{21,22}$, Mathias Schultheis ${ }^{81}$, Axel D. Schwope ${ }^{29}$,

C. G. Scóccola ${ }^{3,4}$, Uros Seljak ${ }^{8,51,63,85}$, Erin Sheldon ${ }^{86}$, Yue Shen ${ }^{44}$, Yiping Shu ${ }^{1}$, Jennifer Simmerer ${ }^{1}$,

Audrey E. Simmons ${ }^{15}$, Ramin A. Skibba ${ }^{45}$, M. F. Skrutskie ${ }^{53}$, A. Slosar ${ }^{86}$, Flavia Sobreira ${ }^{10,25}$, Jennifer S. Sobeck ${ }^{87}$,

Keivan G. Stassun ${ }^{14,88}$, Oliver Steele ${ }^{43}$, Matthias Steinmetz ${ }^{29}$, Michael A. Strauss ${ }^{2,97}$, Alina Streblyanska ${ }^{3}$,

Nao Suzuki ${ }^{8}$, Molly E. C. Swanson ${ }^{44}$, Tomer TaL ${ }^{26}$, Aniruddha R. Thakar ${ }^{69}$, Daniel Thomas ${ }^{43}$,

Benjamin A. Thompson ${ }^{52}$, Jeremy L. Tinker ${ }^{16}$, Rita Tojeiro ${ }^{43}$, Christy A. Tremonti ${ }^{27}$, M. Vargas Magaña ${ }^{7,19}$, Licia Verde ${ }^{38,70}$, Matteo Viel ${ }^{34,35}$, Shailendra K. Vikas ${ }^{89}$, Nicole P. Vogt ${ }^{42}$, David A. Wake ${ }^{26}$, Ji Wang ${ }^{49}$, Benjamin A. Weaver ${ }^{16}$, David H. Weinberg ${ }^{6}$, Benjamin J. Weiner ${ }^{45}$, Andrew A. West ${ }^{90}$, Martin White ${ }^{8}$, John C. Wilson ${ }^{53}$, John P. WisniewsKi ${ }^{5,91}$, W. M. Wood-VASEY ${ }^{89,97}$, Brian Yanny ${ }^{92}$, Christophe YèChe ${ }^{19}$, Donald G. YorK ${ }^{93}$, O. Zamora ${ }^{3,4}$, Gail ZasowsKi ${ }^{53}$, Idit Zehavi ${ }^{56}$, Gong-Bo ZhaO ${ }^{43,94}$, Zheng Zheng ${ }^{1}$, Guangtun Zhu ${ }^{69}$, AND Joel C. ZinN ${ }^{2}$

${ }^{1}$ Department of Physics and Astronomy, University of Utah, Salt Lake City, UT 84112, USA

${ }^{2}$ Department of Astrophysical Sciences, Princeton University, Princeton, NJ 08544, USA

${ }^{3}$ Instituto de Astrofísica de Canarias (IAC), C/Vía Láctea, s/n, E-38200 La Laguna, Tenerife, Spain

${ }^{4}$ Departamento de Astrofísica, Universidad de La Laguna, E-38206 La Laguna, Tenerife, Spain

${ }^{5}$ Department of Astronomy, University of Washington, Box 351580, Seattle, WA 98195, USA

${ }^{6}$ Department of Astronomy, Ohio State University, 140 West 18 th Avenue, Columbus, OH 43210, USA

${ }^{7}$ APC, University of Paris Diderot, CNRS/IN2P3, CEA/IRFU, Observatoire de Paris, Sorbonne Paris Cité, F-75205 Paris, France

${ }^{8}$ Lawrence Berkeley National Laboratory, One Cyclotron Road, Berkeley, CA 94720, USA

${ }^{9}$ Instituto de Física, UFRGS, Caixa Postal 15051, Porto Alegre, RS-91501-970, Brazil

${ }^{10}$ Laboratório Interinstitucional de e-Astronomia, LIneA, Rua Gal. José Cristino 77, Rio de Janeiro, RJ-20921-400, Brazil 


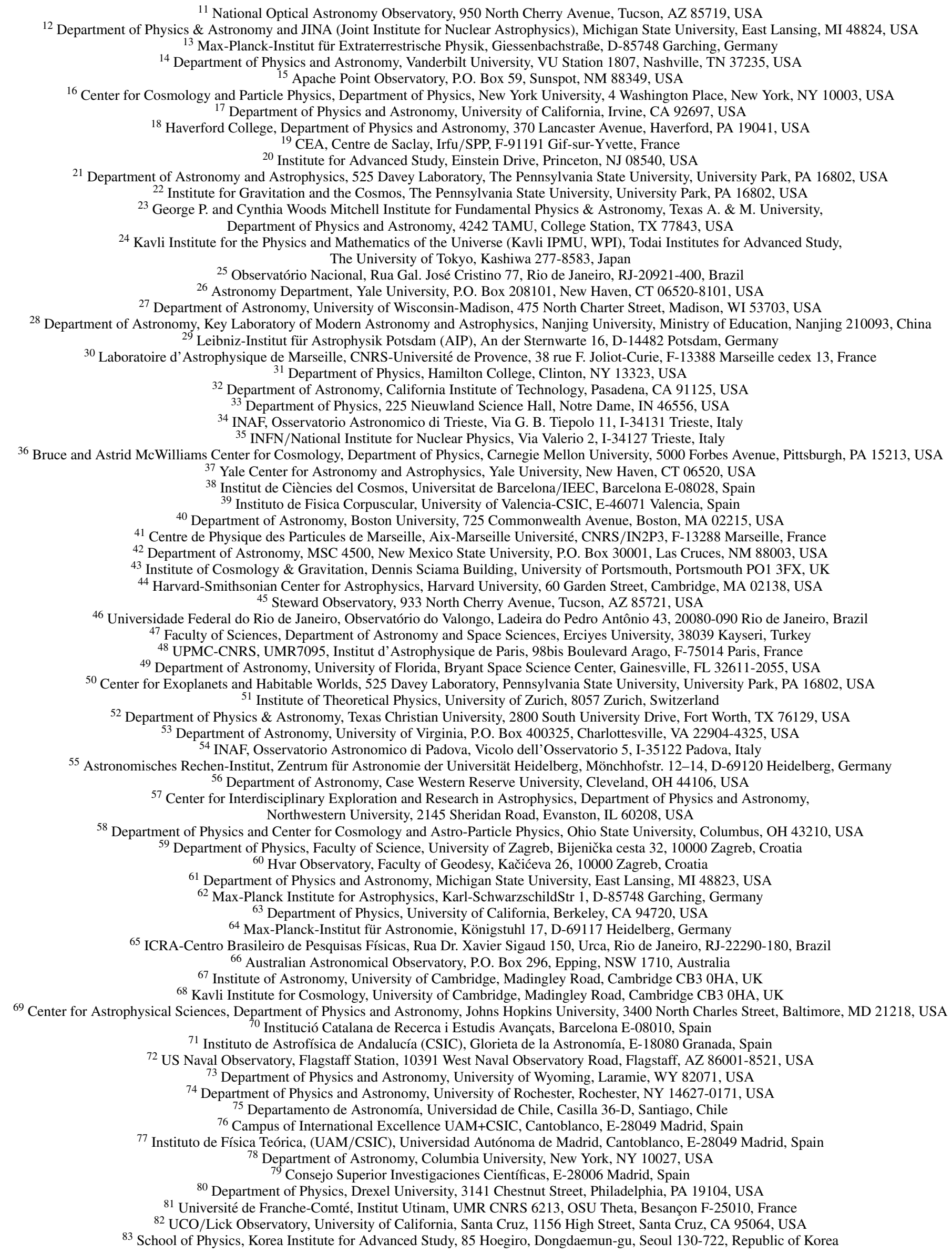




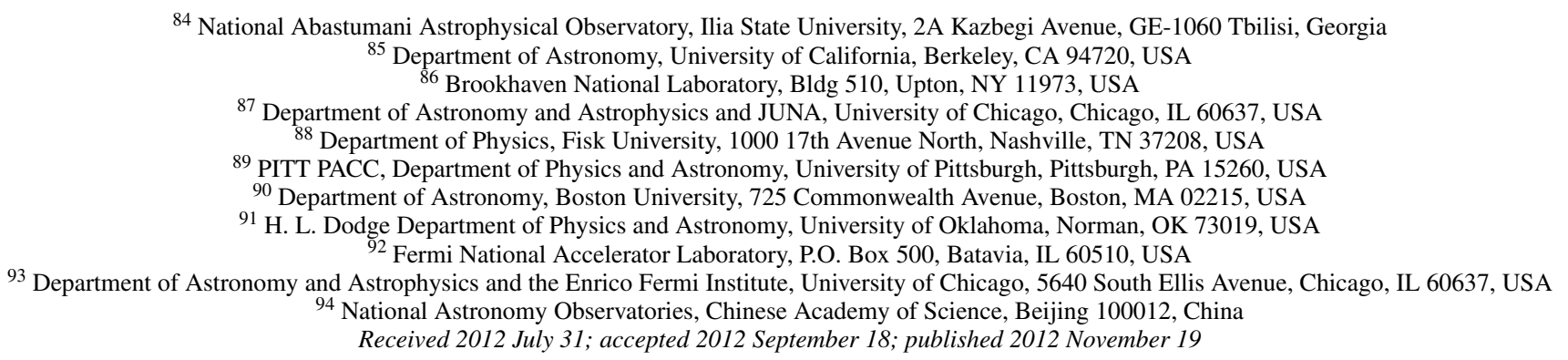

\begin{abstract}
The Sloan Digital Sky Survey III (SDSS-III) presents the first spectroscopic data from the Baryon Oscillation Spectroscopic Survey (BOSS). This ninth data release (DR9) of the SDSS project includes 535,995 new galaxy spectra (median $z \sim 0.52$ ), 102,100 new quasar spectra (median $z \sim 2.32$ ), and 90,897 new stellar spectra, along with the data presented in previous data releases. These spectra were obtained with the new BOSS spectrograph and were taken between 2009 December and 2011 July. In addition, the stellar parameters pipeline, which determines radial velocities, surface temperatures, surface gravities, and metallicities of stars, has been updated and refined with improvements in temperature estimates for stars with $T_{\text {eff }}<5000 \mathrm{~K}$ and in metallicity estimates for stars with $[\mathrm{Fe} / \mathrm{H}]>-0.5$. DR9 includes new stellar parameters for all stars presented in DR8, including stars from SDSS-I and II, as well as those observed as part of the SEGUE-2. The astrometry error introduced in the DR8 imaging catalogs has been corrected in the DR9 data products. The next data release for SDSS-III will be in Summer 2013, which will present the first data from the APOGEE along with another year of data from BOSS, followed by the final SDSS-III data release in 2014 December.
\end{abstract}

Key words: atlases - catalogs - surveys

Online-only material: color figures

\section{INTRODUCTION}

The Sloan Digital Sky Survey III (SDSS-III; Eisenstein et al. 2011) is an extension of the SDSS-I and II projects (York et al. 2000). It uses the dedicated $2.5 \mathrm{~m}$ wide-field Sloan Foundation Telescope (Gunn et al. 2006) at Apache Point Observatory (APO), and fiber-fed multi-object spectrographs to carry out four surveys to study dark energy through observations of distant galaxies and quasars (the Baryon Oscillation Sky Survey, BOSS), to understand the structure of the Milky Way Galaxy (SEGUE-2; and APOGEE), and to search for extrasolar planets (MARVELS). SDSS-III commenced in Fall 2008, and will carry out observations for six years through Summer 2014. The first data release of this phase of SDSS (and the eighth release overall, DR8; Aihara et al. 2011a) was made public in Winter 2011. In addition to all the data from SDSS-I and II (Abazajian et al. 2009), DR8 included additional five-band imaging data over $2500 \mathrm{deg}^{2}$ over the Southern Galactic Cap, as well as stellar spectra from SEGUE-2.

This paper presents the ninth data release (DR9) from SDSS, including all survey-quality data from BOSS gathered through 2011 July. BOSS (Dawson et al. 2012) uses new spectrographs (Smee et al. 2012) to obtain spectra of galaxies with $0.15<z<$ 0.8 and quasars with $2.15<z<3.5$ to measure the scale of the baryon oscillation peak in the correlation function of matter in order to probe the geometry and dynamics of the universe. DR9 includes the first year of BOSS data, and this paper describes the characteristics of these data (summarized in Section 2), with

\footnotetext{
${ }^{95}$ Hubble fellow.

96 National Science Foundation Astronomy and Astrophysics Postdoctoral Fellow.

${ }^{97}$ Corresponding authors.
}

a particular emphasis on how it differs from the spectroscopy carried out in SDSS-I and SDSS-II (Section 3).

The erratum to the DR8 paper (Aihara et al. 2011b) describes a systematic error in the astrometry in the imaging catalogs in DR8. This has now been fixed, as we describe in Section 4.

The SEGUE Stellar Parameters Pipeline (SSPP) fits detailed models to the spectrum of each star, to determine surface temperatures, metallicities, and gravities. It has been continuously improved since its introduction in the sixth data release (DR6; Adelman-McCarthy et al. 2008; see also Lee et al. 2008a). In Section 5, we describe the improvements since DR8 that are incorporated into the DR9 outputs.

Section 6 describes how one can access the DR9 data, and we conclude and outline the planned future data releases in Section 7.

\section{SCOPE OF DR9}

DR9 presents the release of the first 1.5 years of data from the SDSS-III BOSS spectroscopic survey. BOSS started commissioning in early Fall 2009, and began survey-quality observations on the night of 2009 December 5 (UTC-7; MJD 55171). All processed data from that date until the summer telescope shutdown ${ }^{98}$ in 2011 July are included in DR9. All raw data taken by the BOSS spectrograph from the start of commissioning (2009 September) up through and including 2011 July 10 (MJD 55752) are also available as flat files as part of the DR9 release, although the commissioning data are of quite poor quality, and do not always include data from both

\footnotetext{
98 The SDSS telescope pauses science operations during the month-long "monsoon" in July/August in the southwestern United States. This time is used for telescope maintenance and engineering work.
} 
Table 1

Contents of DR9

\begin{tabular}{|c|c|c|}
\hline Imaging $^{\mathrm{a}}$ & Total & Unique $^{\mathrm{b}}$ \\
\hline Area imaged & $31637 \mathrm{deg}^{2}$ & $14555 \mathrm{deg}^{2}$ \\
\hline Cataloged objects & 1231051050 & 469053874 \\
\hline \multicolumn{3}{|c|}{ New BOSS Spectroscopyc } \\
\hline & Total & Unique $^{b}$ \\
\hline Spectroscopic footprint effective area & $\cdots$ & $3275 \mathrm{deg}^{2}$ \\
\hline Plates $^{\mathrm{d}}$ & 831 & 819 \\
\hline Spectra observed ${ }^{\mathrm{e}}$ & 829073 & 763425 \\
\hline Galaxies & 535995 & 493845 \\
\hline CMASS galaxies & 336695 & 309307 \\
\hline LOWZ galaxies & 110427 & 102890 \\
\hline All quasars & 102100 & 93003 \\
\hline Main quasars ${ }^{\mathrm{f}}$ & 85977 & 79570 \\
\hline Main quasars, $2.15<z<3.5^{\mathrm{g}}$ & 59783 & 55047 \\
\hline Ancillary program spectra & 32381 & 28968 \\
\hline Stars & 90897 & 82645 \\
\hline Standard stars & 16905 & 14915 \\
\hline Sky spectra & 78573 & 75850 \\
\hline \multicolumn{3}{|c|}{ All Spectroscopy from SDSS-I/II/III } \\
\hline Total number of spectra & 2674200 & \\
\hline Total number of useful spectra ${ }^{\mathrm{h}}$ & 2598033 & \\
\hline Galaxies & 1457002 & \\
\hline Quasars & 228468 & \\
\hline Stars & 668054 & \\
\hline Sky & 181619 & \\
\hline Unclassified $^{\mathrm{i}}$ & 62890 & \\
\hline
\end{tabular}

Notes.

a These numbers are unchanged since DR8.

b Removing all duplicates and overlaps.

c See Bolton et al. (2012) for full details.

${ }^{\mathrm{d}}$ Twelve plates of the 831 observed plates were re-plugged and re-observed for calibration purposes. Six of the 819 unique plates are different drillings of the same tiling objects.

e This excludes the small fraction of the observations through broken fibers or those that fell out of their holes. There were 831,000 spectra attempted.

f This counts only quasars from the main survey (Section 3.1.2), and does not include those from ancillary programs (Section 3.1.3) or that were used for calibration purposes.

g Quasars with redshifts in the range $2.15<z<3.5$ provide the most signal in the BOSS spectra of the Ly $\alpha$ forest.

h Spectra on good or marginal plates. "Spectrum" refers to a combined set of sub-exposures that define a completed plate. Duplicates are from plates that were observed more than once, or are objects that were observed on overlapping plates.

i Non-sky spectra for which the automated redshift/classification pipeline (Bolton et al. 2012) gave unreliable results, as indicated by the ZWARNING flag.

spectrographs. DR9 also includes the spectroscopic data from SDSS-I/II and SEGUE2; it is unchanged since DR8.

The details of the data included in DR9 are summarized in Table 1, and the footprints of the imaging and spectroscopic data are shown in Figure 1. The imaging data and imaging catalogs are the same as in DR8, with the key update of an improved astrometric solution to correct an error affecting objects at high declinations (Aihara et al. 2011b).

Figure 2 presents the distribution with look-back time of spectroscopically confirmed stars, galaxies, and quasars from BOSS in the DR9 data set. Figure 3 compares these distributions to those of all previous SDSS spectra of galaxies and quasars.

All data released with DR9 are publicly available at http://www.sdss3.org/dr9.
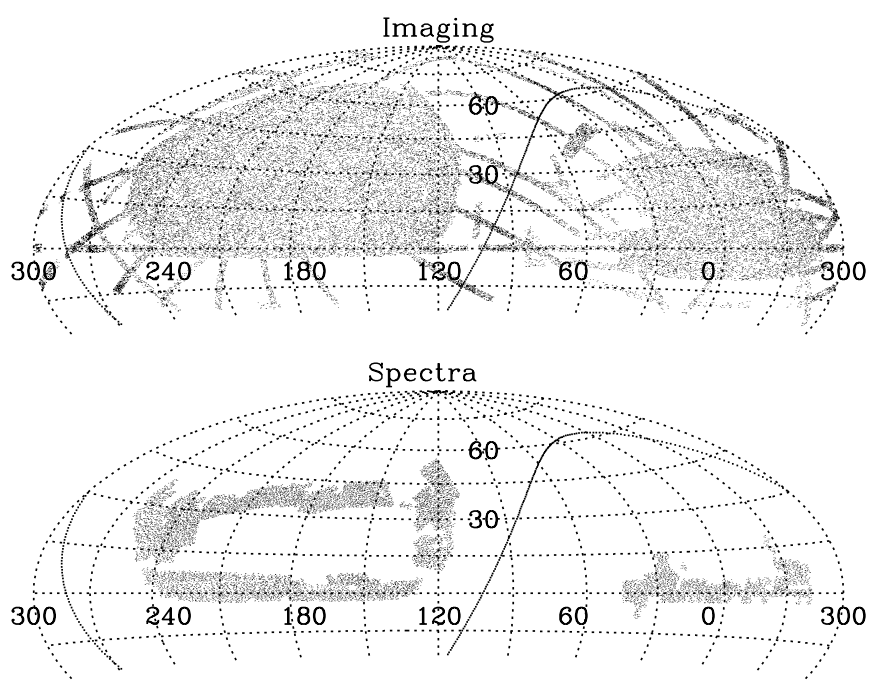

Figure 1. Distribution on the sky of all SDSS imaging (top; $14,555 \mathrm{deg}^{2}$, same as DR8) and BOSS DR9 spectroscopy (bottom; $3275 \mathrm{deg}^{2}$ ) in equatorial coordinates $\left(\alpha=0^{\circ}\right.$ is offset to the right in this projection). The Galactic equatorial plane is shown by the solid line. To make the image for BOSS spectroscopy, we simply plotted a sparse version of the BOSS quasar catalog (Pâris et al. 2012)

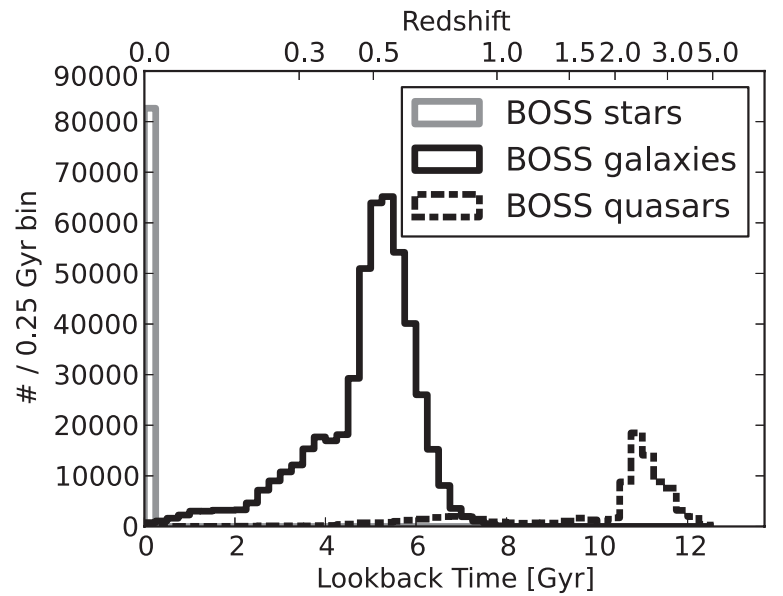

Figure 2. Distribution with look-back time of the 82,645 stars, 493,845 galaxies, and 93,003 quasars with spectra in DR9 BOSS. Look-back time is based on the observed redshift under the assumption of a flat $\Lambda$ CDM cosmology $\left(\Omega_{M}, \Omega_{\Lambda}, h\right)=(0.272,0.728,0.71)$ consistent with the joint cosmological analysis of WMAP7 (Komatsu et al. 2011).

\section{THE BARYON OSCILLATION SPECTROSCOPIC SURVEY}

When the universe was radiation-dominated, sound waves propagated through the radiation-matter fluid at a significant fraction of the speed of light. They slowed dramatically after matter-radiation equality, and were frozen in after recombination. Sound waves emanating from overdensities thus traveled a given distance, roughly 150 comoving Mpc (given standard cosmological parameters) from the initial perturbations; the resulting overdensity gives an excess in the clustering of matter at this scale. This is the origin of the oscillations seen in the power spectrum of the cosmic microwave background (e.g., Komatsu et al. 2011), and was first conclusively seen in the clustering of galaxies from the Two Degree Field Galaxy Redshift Survey (Cole et al. 2005) and the SDSS (Eisenstein et al. 2005). This feature in the galaxy or matter correlation function or power spectrum is a standard ruler; measuring it as a function of 

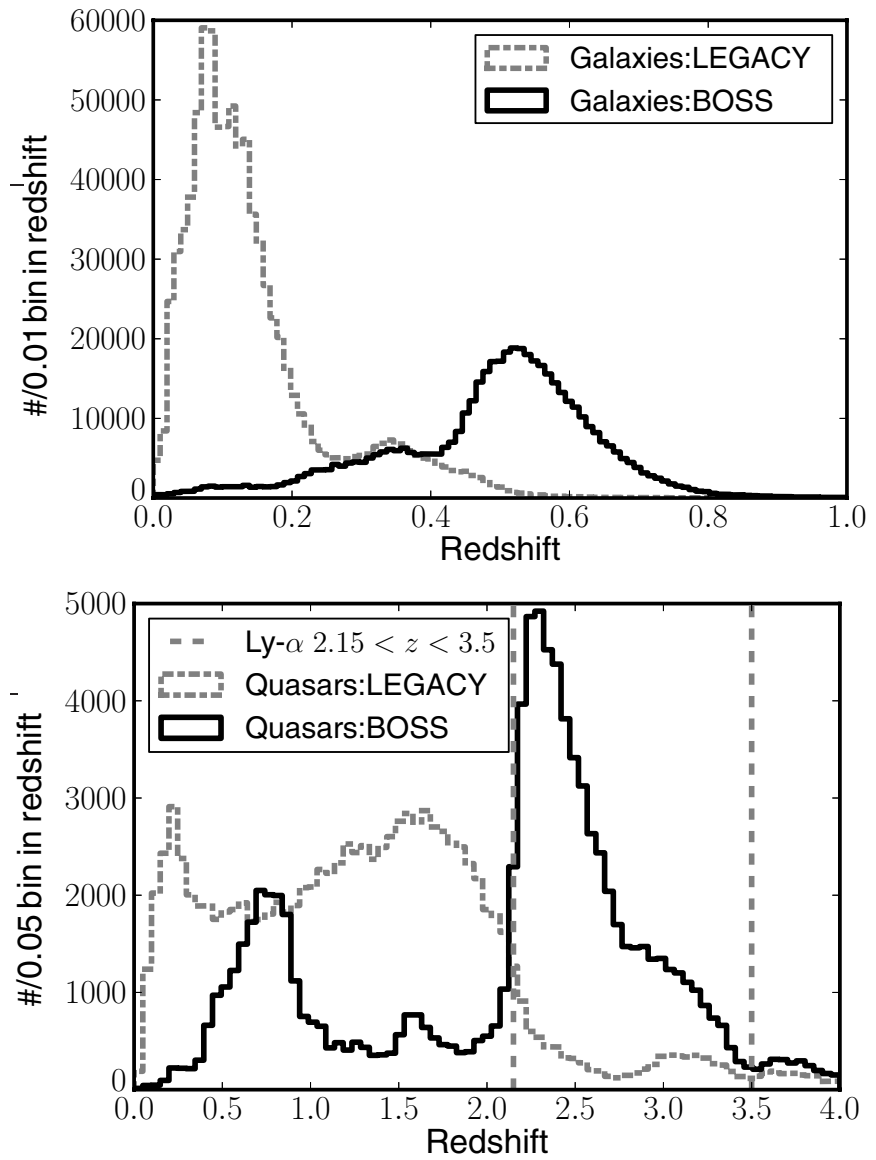

Figure 3. $N(z)$ of BOSS spectra in DR9 compared to that of the SDSS-I/II Legacy spectra for galaxies (top) and quasars (bottom). BOSS' focus on galaxies with $0.4<z<0.6$ and quasars with $z>2.15$ is apparent. The BOSS quasars in the bump from $0.5<z<0.9$ are selected because of a degeneracy in color space between these lower-redshift quasars and those at $z>2.15$.

redshift gives a powerful constraint on cosmological models (e.g., Weinberg et al. 2012).

The initial SDSS detection of the baryon oscillation feature (Eisenstein et al. 2005; see also Tegmark et al. 2006; Percival et al. 2010; Padmanabhan et al. 2012) was based upon a galaxy sample with effective mean redshift $z \sim 0.35$. BOSS aims to measure spectra (and thus redshifts) for a sample of 1.5 million galaxies extending to $z=0.8$ over $10,000 \mathrm{deg}^{2}$, to use the baryon oscillation feature to make a $1 \%$ measurement of the angular diameter distance at $z=0.35$ and a separate uncorrelated $1 \%$ measurement at $z=0.6$. In addition, 150,000 quasars with $z>2.15$ will be observed to measure the clustering of the Ly $\alpha$ forest, and thus to determine the baryon oscillation scale at $z \sim 2.5$, an epoch before dark energy dominated the expansion of the universe.

The samples of galaxies and quasars needed to carry out this program are significantly fainter than those targeted in SDSS-I and SDSS-II (Eisenstein et al. 2001; Strauss et al. 2002; Richards et al. 2002), and have a higher density on the sky. The SDSS spectrographs and supporting infrastructure were extensively rebuilt to increase throughput and observing efficiency, as described in detail in Smee et al. (2012). In particular:

1. The optical fibers, which bring light from the focal plane to the spectrographs, subtended $3^{\prime \prime}$ on the sky in SDSS-I/II. Given the smaller angular size of the higher redshift BOSS galaxy targets, the fibers now subtend $2^{\prime \prime}$.

2. The number of fibers was increased from 640 to 1000 .
3. New high-throughput volume phase holographic (VPH) gratings were installed.

4. The optics have been replaced, with improved throughput.

5. The CCDs were replaced, with improved response at both the blue and red limits.

The resulting spectra are broadly similar to those of SDSS-I/II, but have significantly higher signal-to-noise ratio $(\mathrm{S} / \mathrm{N})$ at a given fiber magnitude. While the resolution as a function of wavelength is similar, the spectral coverage is significantly broader, from $3600 \AA$ to $10400 \AA$. Finally, the target selection algorithms for galaxies (N. Padmanabhan et al. 2013, in preparation) and quasars (Ross et al. 2012) are significantly different from those used in SDSS-I/II, given the rather different scientific goals.

The design of the BOSS survey itself is described in detail in Dawson et al. (2012). First baryon oscillation results from the DR9 galaxy sample may be found in Anderson et al. (2012) and references therein, and the first analysis of the clustering of the Ly $\alpha$ forest from BOSS quasar spectra is found in Slosar et al. (2011).

\subsection{BOSS Main Survey Targets}

There are four broad categories of targets on the BOSS plates: galaxies (Section 3.1.1; see N. Padmanabhan et al. 2013, in preparation), quasars (Section 3.1.2; see Ross et al. 2012), ancillary targets (Section 3.1.3), and standards and calibrations (Dawson et al. 2012).

\subsubsection{Galaxies}

The SDSS-I/II Legacy survey targeted galaxies in two categories: a magnitude-limited sample of galaxies in the $r$ band (Strauss et al. 2002), with a median redshift of $z \sim 0.10$, and a magnitude- and color-limited sample of fainter galaxies designed to select the most luminous red galaxies (LRG) at each redshift (Eisenstein et al. 2001); the LRG sample is approximately volume limited to $z \sim 0.38$, and includes galaxies to $z \sim 0.55$. BOSS aims to measure large-scale clustering of galaxies at higher redshifts and at lower luminosities (to sample the density field at higher space density), and thus targets significantly fainter galaxies.

The galaxy target selection algorithm is described in detail in N. Padmanabhan et al. (2013, in preparation). In brief, it uses the DR8 imaging catalog to select two categories of objects using colors that track the locus of a passively evolving galaxy population with redshift (Maraston et al. 2009). The "LOWZ" subsample, containing about a quarter of all galaxies in BOSS, targets galaxies with $0.15<z<0.4$ with colors similar to LRGs, but with lower luminosity; the space density of LOWZ galaxies is about 2.5 times that of the SDSS-I/II LRG sample. The constant-mass or "CMASS" sample, containing three times more galaxies than LOWZ, is designed to select galaxies with $0.4<z<0.8$. The rest-frame color distribution of this sample is significantly broader than that of the LRG sample, thus CMASS contains a nearly complete sample of massive galaxies above the magnitude limit of the survey. The LOWZ and CMASS samples together give a very roughly volumelimited sample, with space density of order $3 \times 10^{-4}\left(h \mathrm{Mpc}^{-3}\right)$ to $z \sim 0.6$, and a tail to $z \sim 0.8$. In practice, it is somewhat difficult to select objects at $z=0.45$ as the $4000 \AA$ break falls between the $g$ and $r$ bands. The space density of the sample at that redshift is consequently $25 \%$ lower. 
The CMASS sample includes a "SPARSE" extension in color space, to better understand incompleteness in the CMASS sample and to sample a population of fainter, bluer, and less massive galaxies. The galaxies were selected by extending the CMASS color-magnitude cut, and are sub-sampled at 5 galaxies $\mathrm{deg}^{-2}$.

As described in N. Padmanabhan et al. (2013, in preparation), there was an error in the implementation of the LOWZ sample for the early BOSS data (plate numbers 3987 and less); these data should be excluded from any analysis which requires a uniform LOWZ sample.

The BOSS galaxy sample extends about half a magnitude fainter than the SDSS-I/II LRG sample, and thus the S/N of the spectra tend to be lower, despite the higher throughput of the spectrographs. Nevertheless, in DR9 the vast majority of the galaxy targets are confirmed galaxies with confidently measured redshifts: $95.4 \%$ of all CMASS targets and $99.2 \%$ of all LOWZ targets. The $4.6 \%$ of unsuccessful galaxy redshifts for CMASS targets are mostly erroneously targeted red stars. As described in Section 3.3, the $\mathrm{S} / \mathrm{N}$ of the spectra is sufficient that higher-order quantities (stellar masses, velocity dispersions, emission-line properties, and so on) can be measured for most objects.

\subsubsection{Quasars}

The BOSS Quasar Survey uses imaging data from DR8 (Aihara et al. 2011a) to select its main spectroscopic targets. The aim is to observe $z>2.15$ quasars, as for these objects the Ly $\alpha$ forest enters into the spectral coverage of the BOSS spectrographs. This is a challenging task, given the fact that the quasar locus in SDSS color space crosses that of F stars at $z \sim 2.7$ (Fan 1999). Ross et al. (2012) give full details on the BOSS quasar target selection methods that were used. In brief, we implemented and tested a range of methods over the commissioning period and the first year of BOSS spectroscopy (Year One, ending in 2010 July). Quasar targets were selected based on their optical fluxes and colors, and properties in other bands, including radio and near-infrared. Unlike the SDSS-I/II Legacy quasar sample (Richards et al. 2002), the BOSS quasar selection actively selects against quasars with redshifts less than 2.15 (in particular, most ultraviolet excess sources).

As the main science goal of the BOSS quasar sample is to probe the foreground hydrogen in the intergalactic medium, priority was placed on maximizing the surface density of $z>2$ quasars (McDonald \& Eisenstein 2007; McQuinn \& White 2011), rather than aiming for a homogeneous data set. Thus the full target selection is a complicated heterogeneous combination of several methods, using ancillary data sets where available (Ross et al. 2012).

However, to allow statistical studies of quasar physical properties, demographics, and clustering, we defined a subsample (called "CORE" in Ross et al. 2012) that will be uniformly selected throughout BOSS. It uses a single selection algorithm, the extreme deconvolution method (hereafter XDQSO) of Bovy et al. (2011), using single-epoch SDSS photometry. However, we settled on XDQSO only at the end of Year One, and thus the CORE sample in the first year of data is not homogeneous. CORE targets were allocated at $20 \mathrm{deg}^{-2}$, of which $\sim 10-15 \mathrm{deg}^{-2}$ are confirmed spectroscopically to be quasars at $z>2$. An additional 20 targets $\mathrm{deg}^{-2}$ (the "BONUS" sample) were selected using a heterogeneous set of selection criteria to maximize the surface density of high- $z$ quasars; of these, $\sim 5 \mathrm{deg}^{-2}$ are found to be quasars at $z>2$. In Year One, es- pecially in the commissioning period, we increased the number density of targets as we fine-tuned the selection algorithms.

Finally, given the improved throughput of the BOSS spectrographs and extended blue coverage, we re-observed all previously known $z>2.15$ quasars (most of which were discovered by SDSS-I/II; see Schneider et al. 2010) to obtain higher S/N in the Ly $\alpha$ forest.

Approximately half of the quasar targets observed in DR9 were confirmed to be quasars spectroscopically; many of the remaining objects turn out to be F stars.

All quasar targets, plus all objects from other target algorithms spectroscopically identified as quasars via our automated pipeline, have been visually inspected, and both automated pipeline results and these visual redshifts and classifications are provided in DR9. The resulting quasar catalog, together with measurements of broad absorption lines and damped Ly $\alpha$ systems, will be made public in Pâris et al. (2012). A subsample of BOSS quasar spectra suitable for Ly $\alpha$ forest analysis $(z \geqslant 2.15)$ will be described in K.-G. Lee et al. (2012, in preparation), which will provide additional products such as quasar continua, improved noise estimates, and pixel masks.

\subsubsection{BOSS Ancillary Targets}

In addition to the main galaxy and quasar programs, roughly $3.5 \%$ of the BOSS fibers in DR9 were devoted to a series of 25 small ancillary projects, each consisting of a few hundred to a few thousand targets. These programs, described in detail in Appendix A of Dawson et al. (2012), were selected via internal collaboration review, and cover scientific goals ranging from studies of nearby stars to $z>4$ quasars. The ancillary programs allow fibers to be used that would otherwise go unplugged in regions where the principal targets are more sparse than average. These spectra are processed with the same pipeline (D. Schlegel et al. 2012, in preparation; Bolton et al. 2012) as all the other spectra.

A particular focus of many of these ancillary programs is the roughly $220 \mathrm{deg}^{2}$ in the Southern Galactic Cap covered by "Stripe 82" $\left(-1.25<\delta<+1.25,320^{\circ}<\alpha<45^{\circ}\right)$ that was imaged repeatedly in SDSS (Adelman-McCarthy et al. 2008). Using stacked photometry and variability information, for example, the quasar sample on Stripe 82 is particularly complete (e.g., Palanque-Delabrouille et al. 2011).

\subsection{Differences between SDSS-I/II Spectra and SDSS-III BOSS Spectra}

Readers who are familiar with the SDSS-I/II spectra will be able to use the BOSS spectra quickly, since the twin BOSS spectrographs are upgraded versions of the original SDSS-I/II spectrographs, as described above. In addition, the pipelines used to process the BOSS spectra (D. Schlegel et al. 2012, in preparation; Bolton et al. 2012) are improved versions of those used in SDSS-I/II. In this section, we briefly outline the main differences between the BOSS spectra and the SDSSI/II spectra. For more detailed information on the BOSS spectrographs, the reader is referred to Smee et al. (2012), while the BOSS operations are described in Dawson et al. (2012).

The BOSS spectrographs include 1000 fibers in each plate, in comparison with 640 fibers per plate in SDSS-I/II. In addition, the spectral coverage has been increased from 3800-9200 to $3600-10400 \AA$. The dichroic split between the blue and red sides occurs at roughly $6000 \AA$, as it was in SDSS-I/II. The expanded blue coverage means that the $\mathrm{Cd}$ I arc line at $3610.51 \AA$ is now included in the wavelength calibration, enabling a more 


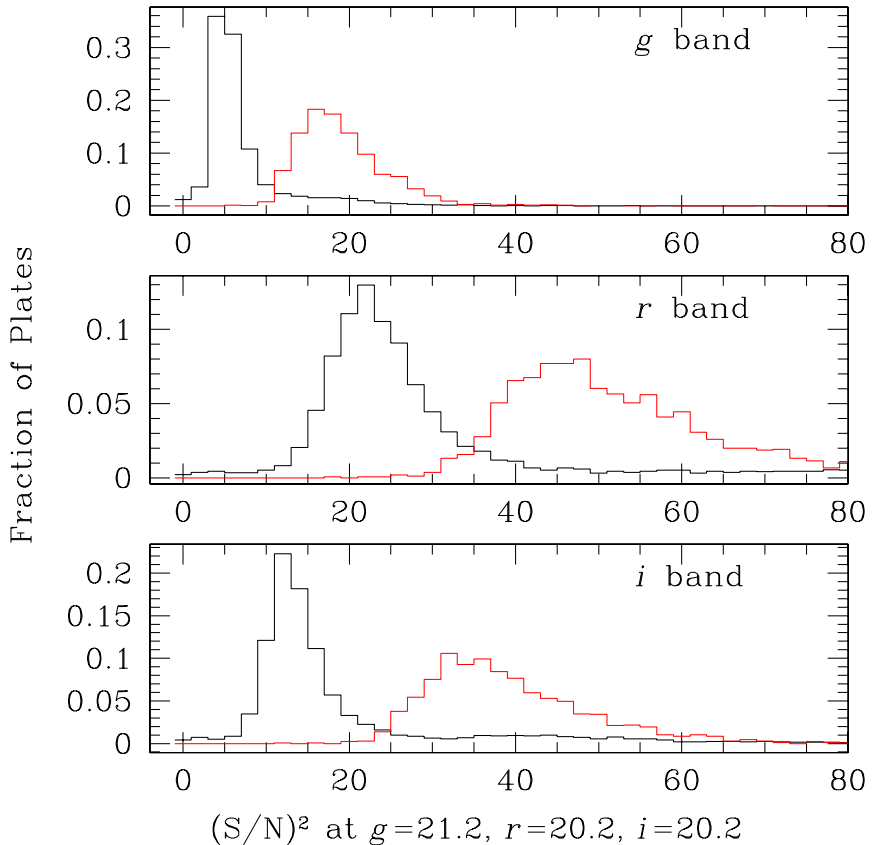

Figure 4. S/N per pixel distribution of DR9 BOSS plates (red), compared with the equivalent for DR7 SDSS-I/II plates (black). The quantity shown is the square of the S/N, measured at a fiducial fiber magnitude. In SDSS-I and SDSS-II, these fiducial magnitudes differ somewhat (and the flux is measured through a $3^{\prime \prime}$ fiber, not a $2^{\prime \prime}$ fiber); these effects have been accounted for in this figure to make a fair comparison.

(A color version of this figure is available in the online journal.)

accurate wavelength solution on the blue end (see the discussion in Adelman-McCarthy et al. 2008). The median resolution of the BOSS spectra remains $R=\lambda / \Delta \lambda \approx 2000$ as in SDSS-I/II, with a similar wavelength dependence (Smee et al. 2012); the resolution ranges from $R \approx 1500$ at $3700 \AA$, to $R \approx 2500$ at $9500 \AA$.

In addition, the diameter of the spectroscopic fibers in BOSS has been decreased in size from $3^{\prime \prime}$ to $2^{\prime \prime}$. While this improves the $\mathrm{S} / \mathrm{N}$ for point-like objects and the smaller galaxies targeted by BOSS due to decreased sky background relative to the source signal, the smaller fiber size affects the spectrophotometry for galaxies, and is more subject to differential chromatic aberration and seeing effects. As in SDSS-I/II, the spectrophotometry is tied to the point-spread function (PSF) photometry of stars on each plate. In SDSS-I/II, the rms scatter between the PSF photometry and synthesized photometry from the calibrated spectra was of order 4\% (Adelman-McCarthy et al. 2008); with BOSS, it is closer to 6\% (Dawson et al. 2012, but see the discussion below about quasar spectrophotometry). The photometric catalog released in DR8 and DR9 (Section 6) provides the $2^{\prime \prime}$ photometry (termed FIBER2MAG) for each object to complement $3^{\prime \prime}$ photometry (FIBERMAG).

The more sensitive CCDs, improved throughput of the VPH gratings, and improved optics have further improved the $\mathrm{S} / \mathrm{N}$ in the BOSS spectra, enabling the targeting of fainter objects. For each plate, the median $\log \mathrm{S} / \mathrm{N}$ per pixel within wavelength regions corresponding to the SDSS imaging bands $g, r$, and $i$ (Fukugita et al. 1996) is tabulated against the corresponding $2^{\prime \prime}$ fiber magnitude. A line of slope 0.3 is fit to this line, and the intercept at the fiducial magnitudes of $g=21.2, r=20.2$, and $i=20.2$ is noted. This quantity is compared for SDSS DR7 and BOSS plates in Figure 4. The median exposure times of BOSS DR9 plates $(1.5 \mathrm{hr})$ are only $70 \%$ longer than those in SDSS-
I/II (0.89 hr), but due to the instrument upgrades, the resulting $(\mathrm{S} / \mathrm{N})^{2}$ values of the BOSS spectra are more than twice those in SDSS-I/II at the same magnitude.

Because one of the stated goals of the BOSS survey is to study the Ly $\alpha$ forest absorption in quasars, efforts have been made to improve the $\mathrm{S} / \mathrm{N}$ at the blue end of the BOSS objects targeted as quasars. In particular, the focal plane of the SDSS telescope was designed to be in focus for BOSS at $\sim 5400 \AA$, whereas the $z \sim 2.5$ Ly $\alpha$ forest lies at $\lambda \lesssim 4000 \AA$, a wavelength that will be out of focus and offset radially due to differential chromatic aberration. To correct for this, we have offset the quasar target fibers in both the radial and axial directions to maximize the throughput at $\lambda \sim 4000 \AA$. The radial offset was implemented by drilling the quasar plug holes at slightly different positions (depending on the assumed hour angle at which the plate will be observed), while in the axial direction we have introduced thin washers to the plug holes on the fiber side of the plates, with thicknesses of 175 and $300 \mu \mathrm{m}$ in the regions spanning 1.02-1.34 deg and 1.34-1.50 deg radially from the plate center, respectively (Dawson et al. 2012). These offsets are tabulated in the ZOFFSET and LAMBDA_EFF flags in the survey data (Section 6).

The current pipeline flux calibration (D. Schlegel et al. 2012, in preparation) does not take these fiber offsets into account, therefore the spectrophotometry of the objects in the quasar targets is biased toward bluer colors, with excess flux relative to the SDSS imaging data at $\lambda<4000 \AA$ and a flux decrement at longer wavelengths (Pâris et al. 2012). We have measured the mean difference between spectrophotometric and imaging magnitudes for those objects targeted as quasars but that turned out to be stars ${ }^{99}$ - the values are $(0.11 \pm 0.24,0.16 \pm 0.29$, $0.24 \pm 0.33)$ mag in $(g, r, i)$. Objects observed at higher airmass show larger offsets.

Quasars targeted solely as part of ancillary programs were not subject to these offsets, and thus their spectrophotometry should show no significant bias. Of course, these objects will have reduced $\mathrm{S} / \mathrm{N}$ in the blue. However, some quasars targeted in ancillary programs were also targets in the main CORE or BONUS samples; these ancillary quasars do have the washer offsets applied (at least after MJD 55441, when the washers were first applied; see Section 3.4 below).

DR9 includes new BOSS observations of objects observed with the previous spectrograph in SDSS-I/II. This includes 4074 galaxies, 16,967 quasars (mostly specifically re-targeted to obtain better Ly $\alpha$ forest measurements), and 7875 stars. The repeated galaxy and star observations confirm that the redshift scales of the two data sets are consistent within a few $\mathrm{km} \mathrm{s}^{-1}$. However, due to an updated set of quasar templates in the BOSS pipeline (Bolton et al. 2012), quasar redshifts are $175 \mathrm{~km} \mathrm{~s}^{-1}$ higher in the median in BOSS than in SDSS-I/II. The limitations of the quasar redshifts in previous data releases were highlighted by Hewett \& Wild (2010) in a reanalysis of DR6 quasar redshifts. While the new templates are designed to more fully represent the range of quasars found, obtaining accurate redshifts remains challenging because of the uncertainty in the relative velocity offsets of different emission lines from the rest frame of the quasar host galaxy system. See Pâris et al. (2012) and Bolton et al. (2012) for a discussion of the details and caveats of quasar redshift determination in DR9.

\footnotetext{
99 We exclude quasars from this comparison to avoid introducing intrinsic quasar variability between the time the photometry and spectroscopy were carried out into the comparison between the two different magnitudes.
} 


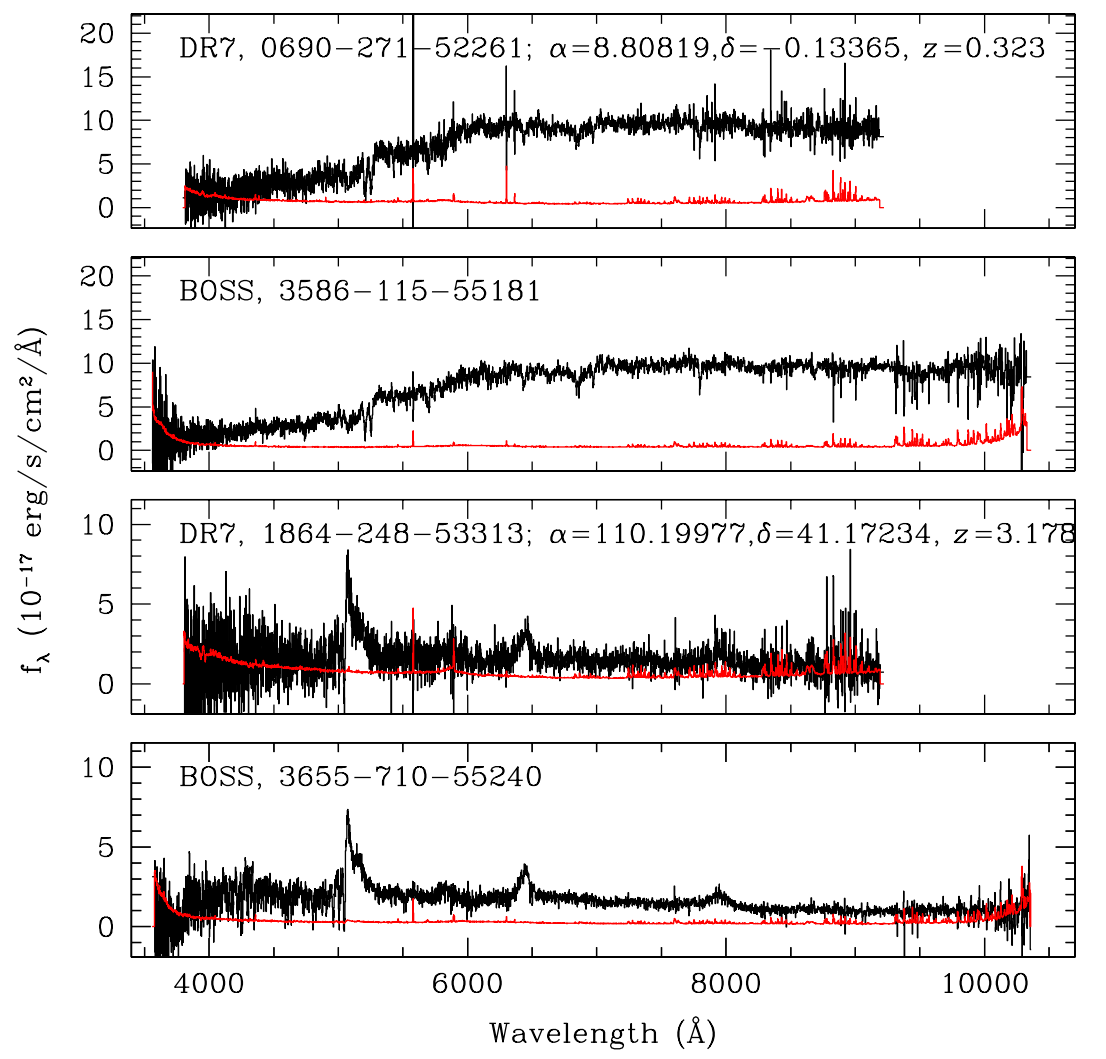

Figure 5. A galaxy (upper panels) and a quasar (lower panels) that were observed in both SDSS-I/II (as released in DR7) and BOSS. These spectra are unsmoothed. In addition to the extended BOSS wavelength coverage from 3600 to $10400 \AA$, the estimated noise per pixel (red line) is lower at every wavelength for the BOSS spectra, particularly at the red and blue ends of the spectrum. This is consistent with the higher $\mathrm{S} / \mathrm{N}$ of the BOSS spectra shown in the distributions in Figure 4. Because the SDSS-I/II spectra are observed through $3^{\prime \prime}$ fibers, while the BOSS spectra use $2^{\prime \prime}$ fibers, one does not expect the galaxy spectra to be identical.

(A color version of this figure is available in the online journal.)

Figure 5 shows spectra of a galaxy and a quasar, observed both with SDSS-I/II and BOSS. This figure illustrates the greater wavelength coverage and the significantly higher $\mathrm{S} / \mathrm{N}$ of the BOSS spectra for observations of the same object.

\subsection{Quantities Derived from Galaxy Spectra}

The spectroscopic pipeline (Bolton et al. 2012) initially classifies all spectra without regard to its imaging data. That is, each object is tested against galaxy, quasar, and stellar templates, regardless of how it was targeted. However, in BOSS, we found that galaxy targets were often incorrectly matched to quasar templates with unphysical fit parameters, e.g., negative coefficients causing a quasar template emission line to fit a galaxy absorption feature. Thus, for galaxy targets in BOSS, the best classification and redshift are selected only from the fits to the galaxy and star templates. The resulting quantities are listed with the suffix _NOQSO in the DR9 database. Results without this template restriction are also made available.

In addition, we have computed a variety of derived quantities from the galaxy spectra following the spectroscopic pipeline, applying stellar population models to derive stellar masses, emission-line fluxes and equivalent widths, stellar and gas kinematics, and velocity dispersions (Chen et al. 2012; Maraston et al. 2012; Thomas et al. 2012).

Each of the stellar population models is applied to all objects that the spectroscopic pipeline calls a galaxy with a reliable and positive definite redshift (i.e., CLASS_NOQSO="GALAXY" and ZWARNING_NOQSO $=0$ and Z_NOQSO $>$ Z_ERR_NOQSO $>0$; see Bolton et al. 2012).
1. Portsmouth spectrophotometric stellar masses (Maraston et al. 2012) are calculated using the BOSS spectroscopic redshift, Z_NOQSO, and $u, g, r, i, z$ photometry by means of broadband spectral energy distribution (SED) fitting of population models. Separate calculations are carried out with a passive template and a star-forming template, and in each case for both Salpeter (1955) and Kroupa (2001) initial mass functions, and for stellar evolution with and without stellar mass loss. Templates are based on Maraston (2005) and Maraston et al. (2009) for the star-forming and passive stellar population models, respectively. In order not to underestimate stellar mass, internal galaxy reddening is not included in the Portsmouth SED fitting procedures used in DR9. Reddening for individual galaxies may, however, be computed via the Portsmouth emission-line flux calculations (see below).

2. Portsmouth emission-line fluxes and equivalent widths, and stellar and gas kinematics (Thomas et al. 2012), are based on the stellar population synthesis models of Maraston \& Strömbäck (2011) applied to BOSS spectra using an adaptation of the publicly available Gas AND Absorption Line Fitting (GANDALF; Sarzi et al. 2006) and penalized PiXel Fitting (pPXF; Cappellari \& Emsellem 2004).

3. Wisconsin stellar masses and velocity dispersions are derived from the optical rest-frame spectral region (3700-5500 Å) using a principal component analysis (PCA) method (Chen et al. 2012). The estimation is based on a library of model spectra generated using the single stellar population models of Bruzual \& Charlot (2003) assuming a Kroupa (2001) initial mass function, and with a broad 
range of star formation histories, metallicities, dust extinctions, and stellar velocity dispersions.

The different stellar mass estimates for BOSS galaxies encompass calculations based on different stellar population models (Bruzual \& Charlot 2003 for Wisconsin, and Maraston 2005 for Portsmouth), different assumptions regarding galaxy star formation histories, reddening, and multiple choices for the initial mass function and stellar mass-loss rates, and each method focuses on a different aspect of the available imaging and spectroscopic data. The Portsmouth SED fitting focuses on broadband colors and BOSS redshifts, the Wisconsin PCA analysis uses the rest-frame $3700-5500 \AA$ stellar continuum region of each galaxy spectrum, and the Portsmouth emissionline fitting focuses on specific regions of the spectrum that contain specific information on gas and stellar kinematics. The array of choices allows consistent comparisons with the literature and future surveys. A detailed comparison between the Portsmouth SED and the Wisconsin spectral PCA calculations can be found in Maraston et al. (2012, Appendix A).

The Galspec product (Kauffmann et al. 2003; Brinchmann et al. 2004; Tremonti et al. 2004) provided by the Max Planck Institute for Astrophysics and the Johns Hopkins University (MPA-JHU) introduced in DR8 is maintained for SDSS-I/II galaxies, but is not available for SDSS-III BOSS spectra. The Portsmouth and Wisconsin stellar population model algorithms are new to DR9 and currently available only for SDSS-III BOSS spectra. However, Chen et al. (2012) and Thomas et al. (2012) each found consistent results between their respective techniques (Wisconsin PCA, and Portsmouth emission-line) and the SDSS-I/II MPA-JHU results for a set of SDSS galaxies from DR7.

\subsection{Changes in BOSS Spectrographs and Survey Strategy}

While commissioning of the BOSS spectrographs was completed in early 2009 December, we continued to make improvements and changes to the spectrographs, the observing system, and the exposure depths. In this section, we outline those changes that affect the DR9 data. The effects on the quality of the resulting spectra due to these changes are subtle, but the reader interested in detailed comparisons of the BOSS data as a function of time should be aware of them.

BOSS observes spectra with 15 minute exposures which are repeated until the summed signal-to-noise squared per pixel, $(\mathrm{S} / \mathrm{N})^{2}$, reaches a given threshold in each of the four spectrograph cameras (B1, B2, R1, R2 for the blue and red arms of spectrographs 1 and 2). A quick-look pipeline runs after each exposure to estimate the accumulated $(\mathrm{S} / \mathrm{N})^{2}$ in near real time and a plate is exposed again until given $(\mathrm{S} / \mathrm{N})^{2}$ thresholds are reached.

For the first year of the survey BOSS conservatively observed a little deeper than was thought to be necessary, with the idea of re-evaluating and updating these $(\mathrm{S} / \mathrm{N})^{2}$ thresholds for future years. After the first year of observations, it became clear that that we were not covering the sky sufficiently quickly to reach our goal of $10,000 \mathrm{deg}^{2}$ by the end of the survey in Summer 2014. BOSS thus conducted a review of the fiducial $(\mathrm{S} / \mathrm{N})^{2}$ thresholds needed to optimize both survey speed and spectroscopic completeness. The decision was made to lower the $(\mathrm{S} / \mathrm{N})^{2}$ thresholds and impose a more restrictive cut on the galaxy surface brightness faint limit. On MJD 55497 the $(\mathrm{S} / \mathrm{N})^{2}$ thresholds were reduced from $>16$ to $>10$ for the blue spectrograph cameras (for $g=22$ ) and from $>26$ to $>22$ for the

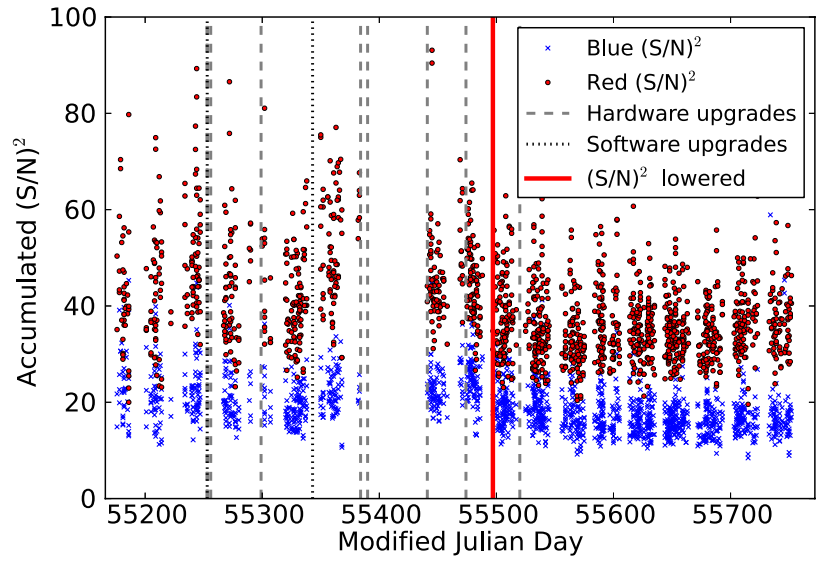

Figure 6. Accumulated signal-to-noise ratio squared per pixel at a fiducial magnitude on each plate, plotted as a function of time for the DR9 BOSS survey data for all completed plates marked as good. The blue $(\mathrm{S} / \mathrm{N})^{2}$ is the average of the signal in blue cameras of the spectrograph for an object with $g=21.2$ ( $2^{\prime \prime}$ fiber magnitude), while the red $(\mathrm{S} / \mathrm{N})^{2}$ is the average of the red cameras of the spectrograph for an object with $r=20.2$. Survey-quality data began at MJD 55171. Changes in survey strategy, hardware, and guider software (Table 2) are indicated with vertical lines. The mean signal-to-noise ratio per plate dropped significantly after the requirements for exposure depths were reduced on MJD 55497 (Section 3.4). The large gap is the 2010 summer shutdown. The smaller gaps are the times of bright moonlight when BOSS does not observe.

(A color version of this figure is available in the online journal.)

red spectrograph cameras (for $i=21$ ). ${ }^{100}$ At the same time, the CMASS target selection limiting magnitude was changed from IFIBER2MAG $<21.7$ to $<21.5$. There is a very slight change in spectroscopic survey completeness after this date. Further details are provided in Section 5 of Dawson et al. (2012).

Improvements to the guider software were made on MJD 55253, leading to better guiding and thus improved throughput. Improvements to the field acquisition software and the efficiency of calibration observations were made on MJD 55343 and resulted in reduced observing overheads and a larger fraction of open-shutter time.

Table 2 summarizes a series of hardware changes that further improved throughput and image quality and reduced scattered light. Figure 6 demonstrates the effect on $\mathrm{S} / \mathrm{N}$ of the hardware and survey strategy changes. This allowed us to reach the fiducial $(\mathrm{S} / \mathrm{N})^{2}$ in the spectra in fewer exposures. Air bubbles had developed in the oil interfaces between the B1 triplet lenses, reducing throughput and causing scattered light. These were replaced on MJD 55520. The triplet lenses for the other spectrograph arms have also been replaced, but only after the 2011 July date that marks the end of DR9. The R2 CCD was replaced on MJD 55298 due to a hardware failure. The R1 and R2 CCD clocking was changed from one- to two-phase for charge collection on MJD 55390. The use of washers to optimize $(\mathrm{S} / \mathrm{N})^{2}$ for quasar targets began on MJD 55441 and was fully implemented for all CORE and BONUS quasar targets starting MJD 55474. Finally, we did two rounds of adjusting the focus of the CCDs in their dewars, further improving the throughput.

\footnotetext{
${ }^{100}$ These values of $(\mathrm{S} / \mathrm{N})^{2}$ are as measured by the quick reductions done of each exposure immediately after it is taken. The full reductions have a moderately higher $(\mathrm{S} / \mathrm{N})^{2}$. The full pipeline also uses a different set of fiducial magnitudes for tracking $(\mathrm{S} / \mathrm{N})^{2}: g=21.2 \mathrm{mag}, r=20.2 \mathrm{mag}$, and $i=20.2$ mag. It is these full pipeline $(\mathrm{S} / \mathrm{N})^{2}$ values that are shown in Figure 4 .
} 
Table 2

BOSS Survey Changes

\begin{tabular}{|c|c|c|}
\hline Date & $\mathrm{MJD}^{\mathrm{a}}$ & Change \\
\hline 2009 Aug 28 & 55071 & Earliest BOSS commissioning data available in SAS \\
\hline 2009 Dec 6 & 55171 & Beginning of survey-quality data \\
\hline 2010 Feb 26 & 55253 & $\begin{array}{l}\text { Installed mask in the central optics to eliminate a secondary light path that } \\
\text { was directly imaged onto the CCD }\end{array}$ \\
\hline 2010 Feb 26 & 55253 & Guider improvements \\
\hline 2010 Feb 26 & 55253 & CCD dewars adjusted for better focus \\
\hline 2010 Mar 1 & 55256 & $\begin{array}{l}\text { Installed a collimator mask to remove light being reflected off of the } \\
\text { slithead and re-imaged onto the CCD }\end{array}$ \\
\hline 2010 Apr 12 & 55299 & R2 CCD replaced \\
\hline 2010 May 28 & 55343 & Field acquisition and calibration efficiency improvements \\
\hline $2010 \mathrm{Jul} 7$ & 55384 & CCD positions adjusted inside dewars for better focus \\
\hline 2010 Jul 13 & 55390 & $\begin{array}{l}\mathrm{R} 1, \mathrm{R} 2 \mathrm{CCD} \text { change from one- to two-phase slightly changed effective } \\
\text { pixel size }\end{array}$ \\
\hline 2010 Sep 2 & 55441 & Washers for quasar targets, some plates \\
\hline 2010 Oct 5 & 55474 & Washers for quasar targets, all plates \\
\hline 2010 Oct 28 & 55497 & Changed $(\mathrm{S} / \mathrm{N})^{2}$ thresholds and target selection \\
\hline 2010 Nov 20 & 55520 & B1 triplet lenses replaced \\
\hline
\end{tabular}

Note. ${ }^{a}$ All data taken on and after the given MJD include the respective change.

\section{FIXED AND IMPROVED ASTROMETRY}

The DR8 imaging suffered from several errors in the astrometric calibration, as described in an erratum published shortly after the DR8 release (Aihara et al. 2011b). ${ }^{101}$ These errors have been corrected in DR9, and the resulting astrometry and proper motions are improved relative to both DR7 and DR8. ${ }^{102}$

The issues with the DR8 astrometry were, in brief:

1. Northward of $+41^{\circ}$ declination there was an offset of 250 mas introduced by switching from the Second US Naval Observatory CCD Astrograph Catalog (UCAC2; Zacharias et al. 2004) to the United States Naval Observatory (USNO)-B catalog (Monet et al. 2003) at this declination.

2. Color terms were not used in calculating CCD position to sky position, introducing systematic errors of 10-20 mas.

3 . UCAC2 proper motions were not applied correctly, introducing further errors of order 5-10 mas.

4. Stellar positions were always measured in the $r$-band photometry, even if the $r$ band was saturated or had a lower $\mathrm{S} / \mathrm{N}$ detection than other filters. For faint objects this increases the statistical uncertainty for the measurement, but for $r$-band saturated objects the difference can be as much as 100 mas between using $r$-band positions and those in non-saturated filters.

All of these issues have been corrected for DR9. The discovery of the mistakes in DR8 prompted the development of a new set of astrometric quality-assurance metrics that are fully described in the SDSS-III DR9 data model. ${ }^{103}$

With these problems corrected, the DR9 astrometry fixes errors in both DR8 and DR7. In particular, DR7 contained very large errors in a handful of runs $(3358,4829,5960,6074$, and 6162) that are corrected in DR9 (the most prominent of these is the black and white arc in the upper central region of the top panels of Figure 7).

\footnotetext{
101 These errors do not appear in the DR7 and earlier releases.

102 While the FITS images distributed as part of the Science Archive Server, http://data.sdss3.org/sas/dr9/, are identical to DR8 on a pixel-by-pixel basis, the FITS image metadata (in particular, the World Coordinate System headers) have been changed to match the revised astrometry in DR9.

$103 \mathrm{http}$ ://data.sdss3.org/datamodel
}

Proper motions in DR9 are similarly improved relative to DR8 and DR7. As Figure 7 shows, they are mostly unchanged in the mean at high Galactic latitudes. However, the corrected color terms in the astrometry have fixed a small fraction of objects with outlying proper motions in the DR9 relative to DR8 (this error did not affect DR7 or earlier). Furthermore, at low Galactic latitudes DR7 had some large offsets caused by star-galaxy separation errors. Proper motions are measured with respect to a reference frame of stationary galaxies, so stellar contamination in the galaxy sample can systematically affect the proper-motion estimates. In DR7, errors in star-galaxy separation (in particular in photometric rerun 648) caused the galaxy sample to have significant stellar contamination, leading to systematic errors in the proper motions. DR9 fixes this problem.

The proper motions can be independently tested by looking at the proper motions of photometrically identified low-redshift quasars, which are easy to select and should have zero proper motions. Figure 8 shows the proper motions of the low-redshift quasars as selected by Bovy et al. (2011). These show very little systematic offset from zero, except for a small shift in $\mu_{\delta}$ at low declination. This offset is further described by Bond et al. (2010) in the context of the DR7 proper motions, and could be due to small differential refraction correction issues in USNO-B for these very blue objects (and is therefore likely not relevant to the proper motions of typical stars).

\section{IMPROVEMENTS IN THE SEGUE STELLAR PARAMETER PIPELINE FOR DR9}

The SSPP (Lee et al. 2008a, 2008b; Allende Prieto et al. 2008; Smolinski et al. 2011) utilizes multiple approaches to estimate effective temperature $\left(T_{\text {eff }}\right)$, surface gravity ( $\log g$ ), and metallicity $([\mathrm{Fe} / \mathrm{H}])$ from stellar spectra. Each method is optimized for a certain range of stellar color $(g-r)$ and $\mathrm{S} / \mathrm{N}$, and is measured over a range of wavelengths determined to deliver the best estimate of each parameter. The SSPP is designed to obtain reliable results for stars targeted as part of the SDSS-II SEGUE and SDSS-III SEGUE-2 surveys (C. Rockosi et al. 2012, in preparation). With each SDSS data release the SSPP has been refined and modified to provide more accurate estimates of the stellar atmospheric parameters. Here we briefly 

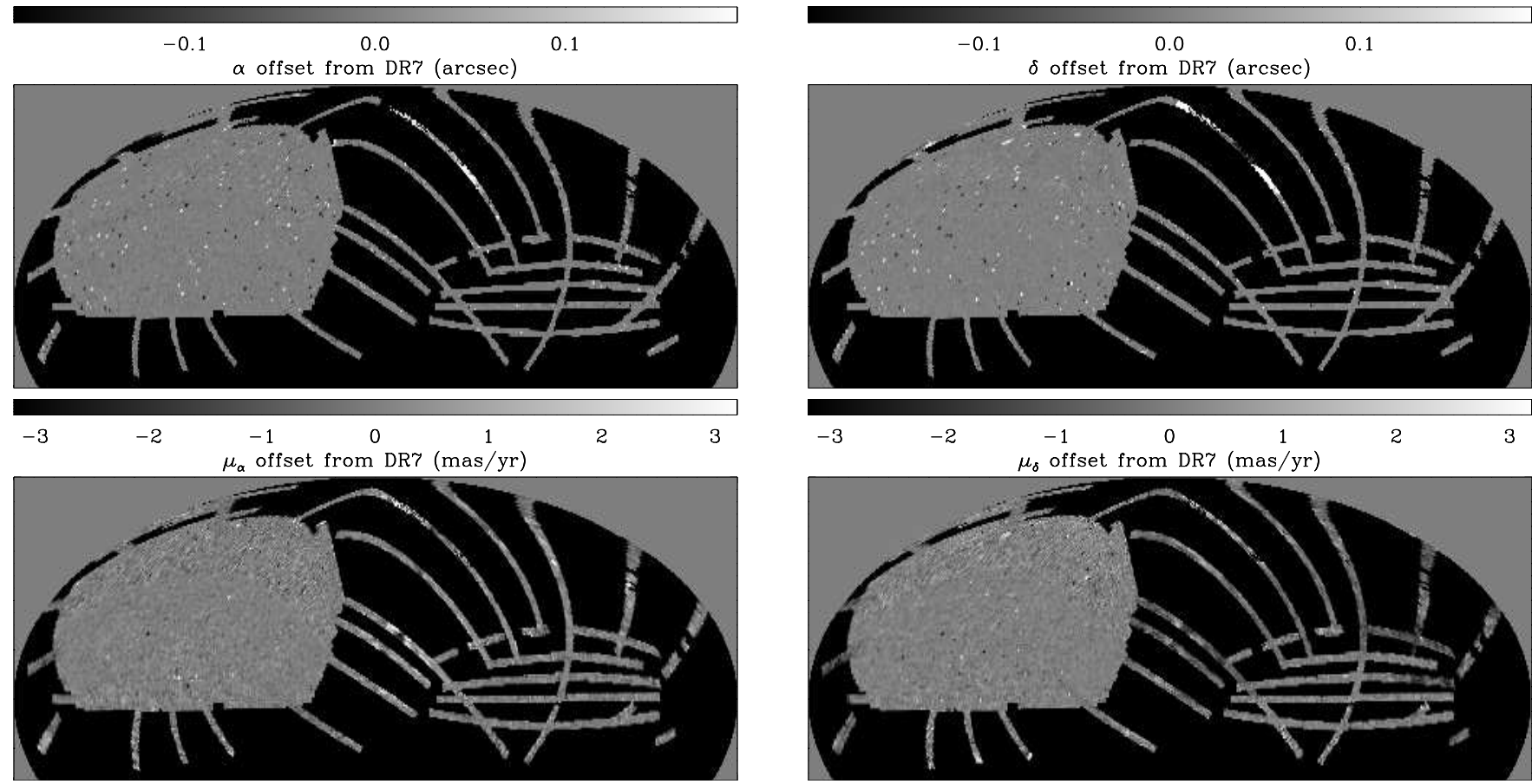

Figure 7. Astrometric and proper-motion comparison of DR9 to DR7, plotted in equatorial coordinates. The top row shows the difference in right ascension (left) and declination (right) of objects matched between the two data releases, and the bottom row shows the differences in their proper motions. In the top row, the DR7 and DR9 astrometry agree over most of the area, with the exception of a handful of spots, all due to errors in the DR7 astrometry. In the bottom row, DR7 and DR9 proper motions agree over virtually all of the high Galactic latitude areas. At low Galactic latitudes there are substantial shifts, caused by errors in DR7 due to mistakes in star/galaxy separation affecting the proper-motion estimates.
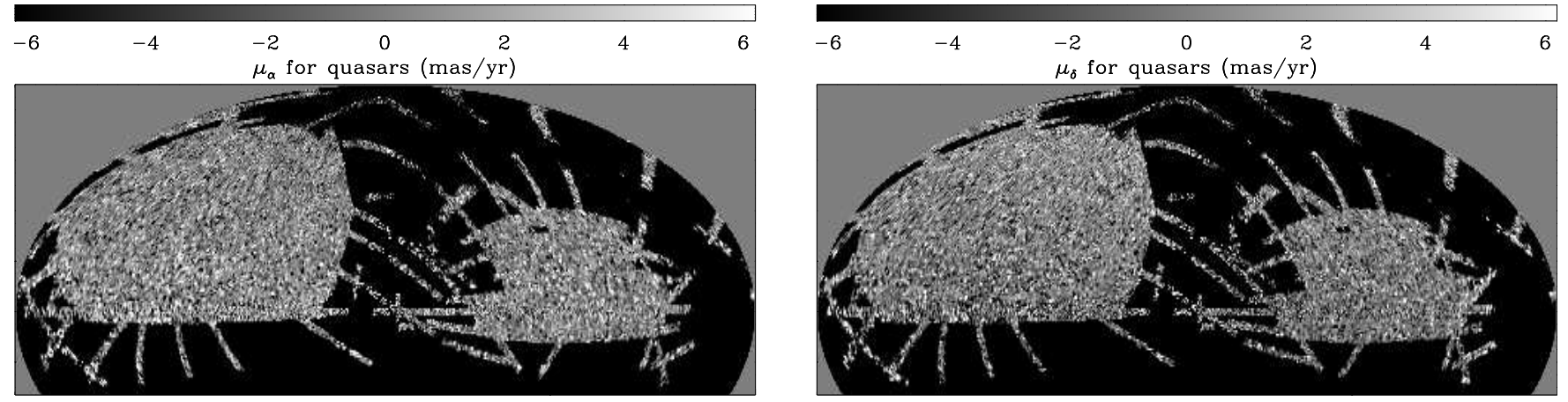

Figure 8. DR9 proper motions of photometrically selected $z<2$ quasars (as classified by Bovy et al. 2011). These motions are nearly consistent with zero, with a slight offset in $\mu_{\delta}$ at low declination, possibly due to errors in differential refraction corrections in USNO-B for these very blue objects (see Bond et al. 2010).

highlight major changes and improvements made since the DR8 public release that are used for the DR9 data.

A sample of 126 high-resolution spectra of SDSS/SEGUE stars, taken with Keck, Subaru, the Hobby-Eberly Telescope, and the Very Large Telescope, have been analyzed in a homogeneous fashion, and a new set of stellar parameters were obtained from this analysis (Allende Prieto et al. 2008; Smolinski et al. 2011). The sample covers $4000 \mathrm{~K}<T_{\text {eff }}<7000 \mathrm{~K}$, $0.0<\log g<5.0$, and $-4.0<[\mathrm{Fe} / \mathrm{H}]<+0.5$. However, this data set contains no metal-poor $([\mathrm{Fe} / \mathrm{H}]<-2.5)$ dwarfs or metal-rich $([\mathrm{Fe} / \mathrm{H}]>0.0)$ giants. Additional information on this high-resolution sample can be found in Allende Prieto et al. (2008) and Smolinski et al. (2011).

The individual methods in the SSPP, in particular estimates of surface gravity and metallicity, have been thoroughly recalibrated based on these new data. The SSPP also adopts a much-improved color $(g-r)$-temperature relation, the InfraRed
Flux Method (IRFM) as described by Casagrande et al. (2010). Each SSPP temperature estimate was re-scaled to match the IRFM temperature estimate. This technique particularly improves the temperature estimates for cool stars $\left(T_{\mathrm{eff}}<5000 \mathrm{~K}\right)$.

Figure 9 shows the results of the comparisons of the SSPP parameters with the IRFM for temperature, and the high-resolution analysis for gravity and metallicity. Implementation of a grid of synthetic spectra with microturbulences that vary appropriately with surface gravity also yields improved estimates of metallicity for metal-rich stars $([\mathrm{Fe} / \mathrm{H}]>-0.5)$.

A parameter comparison from a sample of about 9000 multiply observed stellar spectra in SEGUE provides the basis for an estimate of the internal uncertainties of the SSPP: $\sim 50 \mathrm{~K}$ for $T_{\text {eff }}, \sim 0.12 \mathrm{dex}$ for $\log g$, and $\sim 0.10 \mathrm{dex}$ for $[\mathrm{Fe} / \mathrm{H}]$ for a typical G-type dwarf or redder stars in the color range of $0.4<g-r<1.3$ with $\mathrm{S} / \mathrm{N}$ per pixel $=30$. These errors increase to $\sim 80 \mathrm{~K}, 0.30 \mathrm{dex}$, and $0.25 \mathrm{dex}$ for $T_{\text {eff }}, \log g$, and 

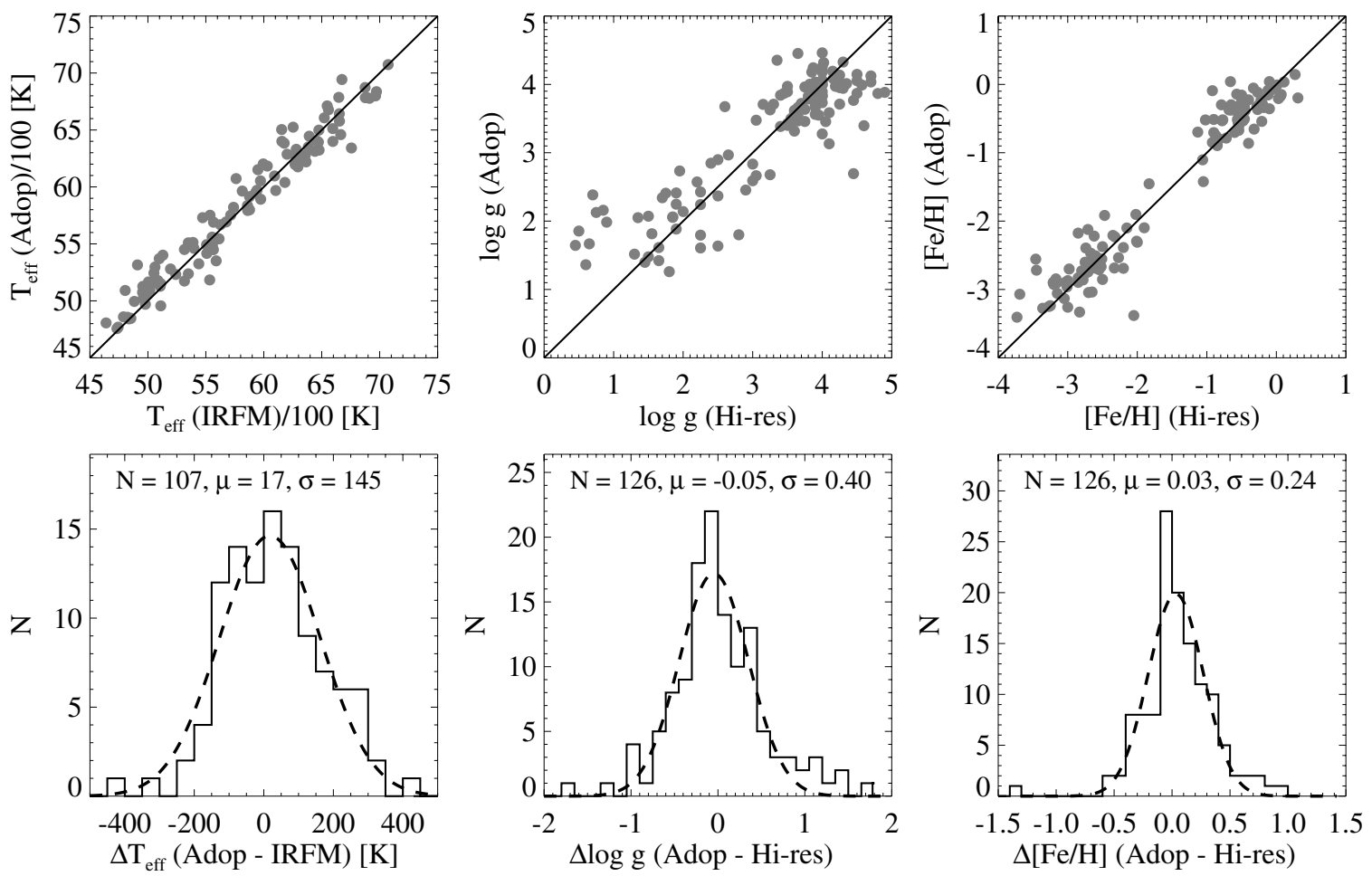

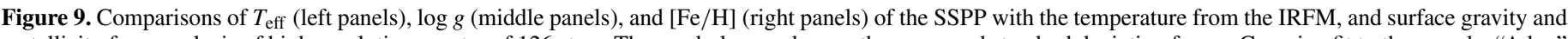

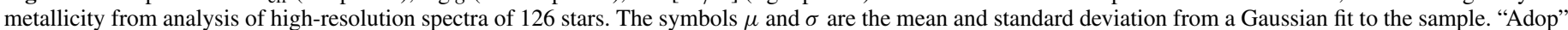

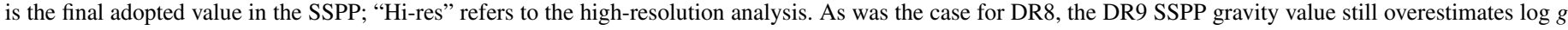

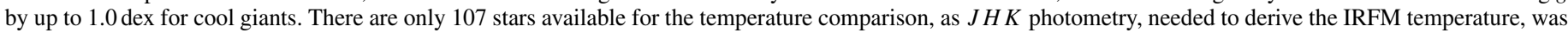
unavailable for 19 stars.

$[\mathrm{Fe} / \mathrm{H}]$, respectively, for stars with $-0.3<g-r<0.2$, $[\mathrm{Fe} / \mathrm{H}]<-2.0$, and $\mathrm{S} / \mathrm{N}<15$.

A comparison with the DR8 parameters for stars from SEGUE-1 indicates that the DR9 average $T_{\text {eff }}$ is higher by $\sim 60 \mathrm{~K}$, the DR9 $\log g$ is lower by $\sim 0.2 \mathrm{dex}$, and the metallicity does not change significantly, although these values vary with spectral type and spectral $\mathrm{S} / \mathrm{N}$.

These new SSPP results are made available for all stars in SDSS-I/II, including those of SEGUE-1 (Yanny et al. 2009), and the SEGUE-2 stars in SDSS-III. SSPP measurements are not currently available for the stars observed as part of BOSS, although we plan to include that in future data releases.

\section{DATA DISTRIBUTION}

All Data Release 9 data are available through data access tools linked from the DR9 Web site. ${ }^{104}$ The data are stored both as flat files in the Science Archive Server (SAS), ${ }^{105}$ and as a searchable database in the Catalog Archive Server (CAS). A number of different interfaces are available, each designed to accomplish a specific task: (1) color images of regions of the sky in JPEG format (based on the $g, r$, and $i$ images; see Lupton et al. 2004) can be viewed in a Web browser with the SkyServer Navigate tool; (2) FITS images can be downloaded through the SAS; (3) complete catalog information (astrometry, photometry, etc.) of any imaging object can be viewed through the SkyServer Explore tool; and (4) FITS files of the spectra can be downloaded through the SAS.

\footnotetext{
104 http://www.sdss3.org/dr9

105 The Science Archive Server (SAS) is the SDSS-III equivalent of the SDSS-I/II Data Archive Server (DAS).
}

In addition, a number of catalog search tools are available through the SkyServer interface to the CAS, each of which returns catalog data for objects that match supplied criteria. For more advanced queries, a powerful and flexible catalog search Web site called "CasJobs" allows users to create their own personalized data sets and then to modify or graph their data.

The DR9 Web site also features data access tutorials, a glossary of SDSS terms, and detailed documentation about algorithms used to process the imaging and spectroscopic data and select spectroscopic targets.

Imaging and spectroscopic data from all prior data releases are also available through DR9 data access tools.

\section{CONCLUSIONS}

The SDSS-III Data Release 9 presents the first data from the BOSS survey, with $\sim 102,000$ new quasar spectra, $\sim 91,000$ new stellar spectra, and $\sim 536,000$ new galaxy spectra. The astrometry has been improved since DR8, and the stellar properties for SEGUE-I/II and SDSS-I/II stars have been updated.

These data are already sufficient for cosmological analyses of large-scale structure, investigations of the structure of the Milky Way, measurements of quasar physics, clustering, and demographics, and countless other science investigations. We invite the larger scientific community to investigate and explore this new data set.

The SDSS-III project will present two more public data releases. DR10, in Summer 2013, will include the first data from the APOGEE survey and another year of BOSS data. DR11 will be an internal release only, as a public release would 
occur only six months before the final public data release for SDSS-III, DR12, which will be released in 2014 December and will contain all of the data taken during the six years of the project.

Funding for SDSS-III has been provided by the Alfred P. Sloan Foundation, the Participating Institutions, the National Science Foundation, and the U.S. Department of Energy Office of Science. The SDSS-III Web site is http://www.sdss3.org/.

SDSS-III is managed by the Astrophysical Research Consortium for the Participating Institutions of the SDSS-III Collaboration including the University of Arizona, the Brazilian Participation Group, Brookhaven National Laboratory, University of Cambridge, Carnegie Mellon University, University of Florida, the French Participation Group, the German Participation Group, Harvard University, the Instituto de Astrofisica de Canarias, the Michigan State/Notre Dame/JINA Participation Group, Johns Hopkins University, Lawrence Berkeley National Laboratory, Max Planck Institute for Astrophysics, Max Planck Institute for Extraterrestrial Physics, New Mexico State University, New York University, Ohio State University, Pennsylvania State University, University of Portsmouth, Princeton University, the Spanish Participation Group, University of Tokyo, University of Utah, Vanderbilt University, University of Virginia, University of Washington, and Yale University.

\section{REFERENCES}

Abazajian, K. N., Adelman-McCarthy, J. K., Agüeros, M. A., et al. 2009, ApJS, 182,543

Adelman-McCarthy, J. K., Agüeros, M. A., Allam, S. S., et al. 2008, ApJS, 175, 297

Aihara, H., Allende Prieto, C., An, D., et al. 2011a, ApJS, 193, 29

Aihara, H., Allende Prieto, C., An, D., et al. 2011b, ApJS, 195, 26

Allende Prieto, C., Sivarani, T., Beers, T. C., et al. 2008, AJ, 136, 2070

Anderson, L., Aubourg, E., Bailey, S., et al. 2012, arXiv:1203.6594

Bolton, A. S., Schlegel, D. J., Aubourg, E., et al. 2012, AJ, 144, 144

Bond, N. A., Ivezić, Ž., Sesar, B., et al. 2010, ApJ, 716, 1

Bovy, J., Hennawi, J. F., Hogg, D. W., et al. 2011, ApJ, 729, 141

Brinchmann, J., Charlot, S., White, S. D. M., et al. 2004, MNRAS, 351, 1151

Bruzual, G., \& Charlot, S. 2003, MNRAS, 344, 1000

Cappellari, M., \& Emsellem, E. 2004, PASP, 116, 138

Casagrande, L., Ramírez, I., Meléndez, J., Bessell, M., \& Asplund, M. 2010, A\&A, 512, A54
Chen, Y.-M., Kauffmann, G., Tremonti, C. A., et al. 2012, MNRAS, 421, 314

Cole, S., Percival, W. J., Peacock, J. A., et al. 2005, MNRAS, 362, 505

Dawson, K. S., Schlegel, D. J., Ahn, C. P., et al. 2012, arXiv:1208.0022

Eisenstein, D. J., Annis, J., Gunn, J. E., et al. 2001, AJ, 122, 2267

Eisenstein, D. J., Weinberg, D. H., Agol, E., et al. 2011, AJ, 142, 72

Eisenstein, D. J., Zehavi, I., Hogg, D. W., et al. 2005, ApJ, 633, 560

Fan, X. 1999, AJ, 117, 2528

Fukugita, M., Ichikawa, T., Gunn, J. E., et al. 1996, AJ, 111, 1748

Gunn, J. E., Siegmund, W. A., Mannery, E. J., et al. 2006, AJ, 131, 2332

Hewett, P. C., \& Wild, V. 2010, MNRAS, 405, 2302

Kauffmann, G., Heckman, T. M., White, S. D. M., et al. 2003, MNRAS, 341,33

Komatsu, E., Smith, K. M., Dunkley, J., et al. 2011, ApJS, 192, 18

Kroupa, P. 2001, MNRAS, 322, 231

Lee, Y. S., Beers, T. C., Sivarani, T., et al. 2008a, AJ, 136, 2022

Lee, Y. S., Beers, T. C., Sivarani, T., et al. 2008b, AJ, 136, 2050

Lupton, R., Blanton, M. R., Fekete, G., et al. 2004, PASP, 116, 133

Maraston, C. 2005, MNRAS, 362, 799

Maraston, C., Pforr, J., Henriques, B. M., et al. 2012, arXiv:1207.6114

Maraston, C., \& Strömbäck, G. 2011, MNRAS, 418, 2785

Maraston, C., Strömbäck, G., Thomas, D., Wake, D. A., \& Nichol, R. C. 2009, MNRAS, 394, L107

McDonald, P., \& Eisenstein, D. J. 2007, Phys. Rev. D, 76, 063009

McQuinn, M., \& White, M. 2011, MNRAS, 415, 2257

Monet, D. G., Levine, S. E., Canzian, B., et al. 2003, AJ, 125, 984

Padmanabhan, N., Xu, X., Eisenstein, D. J., et al. 2012, MNRAS, in press (arXiv:1202.0090)

Palanque-Delabrouille, N., Yeche, Ch., Myers, A. D., et al. 2011, A\&A, 530, A122

Pâris, I., Petitjean, P., Aubourg, E., et al. 2012, A\&A, in press (arXiv:1210.5166)

Percival, W. J., Reid, B. A., Eisenstein, D. J., et al. 2010, MNRAS, 401, 2148

Richards, G. T., Fan, X., Newberg, H. J., et al. 2002, AJ, 123, 2945

Ross, N. P., Myers, A. D., Sheldon, E. S., et al. 2012, ApJS, 199, 3

Salpeter, E. E. 1955, ApJ, 121, 161

Sarzi, M., Falcón-Barroso, J., Davies, R. L., et al. 2006, MNRAS, 366, 1151

Schneider, D. P., Richards, G. T., Hall, P. B., et al. 2010, AJ, 139, 2360

Slosar, A., Font-Ribera, A., Pieri, M. M., et al. 2011, J. Cosmol. Astropart. Phys., JCAP09(2011)001

Smee, S., Gunn, J. E., Uomoto, A., et al. 2012, arXiv:1208.2233

Smolinski, J. P., Lee, Y. S., Beers, T. C., et al. 2011, AJ, 141, 89

Strauss, M. A., Weinberg, D. H., Lupton, R. H., et al. 2002, AJ, 124, 1810

Tegmark, M., Eisenstein, D. J., Strauss, M. A., et al. 2006, Phys. Rev. D, 74, 123507

Thomas, D., Steele, O., Maraston, C., et al. 2012, (arXiv:1207.6115)

Tremonti, C. A., Heckman, T. M., Kauffmann, G., et al. 2004, ApJ, 613, 898

Weinberg, D. H., Mortonson, M. J., Eisenstein, D. J., et al. 2012, arXiv: 1201.2434

Yanny, B., Rockosi, C., Newberg, H. J., et al. 2009, AJ, 137, 4377

York, D. G., Adelman, J., Anderson, J. E., Jr., et al. 2000, AJ, 120, 1579

Zacharias, N., Urban, S. E., Zacharias, M. I., et al. 2004, AJ, 127, 3043 